\title{
EIGENOSCILLATIONS OF MECHANICAL SYSTEMS WITH BOUNDARY CONDITIONS CONTAINING THE FREQUENCY
}

\author{
BY \\ B. P. BELINSKIY AND J. P. DAUER \\ Dept. of Mathematics, University of Tennessee at Chattanooga
}

\begin{abstract}
The problem of eigenoscillations of beam-mass systems is investigated and four examples are developed. For such systems the corresponding Sturm-Liouville problems contain the eigenvalue parameter in the boundary conditions. It is shown that the eigenfunctions for the systems considered form a basis of the appropriate Hilbert space. Rayleigh-Ritz formulas are also developed. Some lower bound estimations for eigenfrequencies are also found.
\end{abstract}

1. Introduction. As is well known, the set of eigenfunctions of a positive selfadjoint second-order differential operator on $L^{2}[0, d]$ forms a basis of the space involved [1, p. 167]. There exists diverse literature [1] devoted to the problem of determining when a system of eigenfunctions forms a basis for the appropriate Hilbert space. The goal of this work is to contribute to this eigenfunction problem for certain mechanical systems that are defined by a Sturm-Liouville problem with an eigenvalue appearing in the boundary conditions. The intention of this paper is to show how known methods and results from the theory of operators on a Hilbert space can be applied to a class of eigenoscillation problems in mechanics. Appropriate subspaces of the Sobolev spaces $H^{k}[0, d], k=1,2$ are used with alternative inner products that are equivalent to the Sobolev inner product and yet that allow the operator to be selfadjoint on the space. The examples presented are taken from the recent engineering and physics literature (see, for example, [2]-[5]) and, it is thought, progressively show the applicability of these operator-theoretic methods to such eigenoscillation problems.

The basic mechanical system considered is that of free oscillations of a string with fixed end points. The vertical displacement $u(z), z \in[0, d]$, is the solution of the SturmLiouville problem

$$
T u^{\prime \prime}+\omega^{2} \mu u=0, \quad z \in(0, d), \quad u(0)=u(d)=0 .
$$

Received January 12, 1996.

1991 Mathematics Subject Classification. Primary 34L10, 34L15; Secondary 46E35, 73D30.

Key words and phrases. Eigenoscillations, beam-mass systems, Sturm-Liouville problem, operator polynomial, basis. 
Here $T$ and $\mu$ are the parameters of a string of length $d>0$, and the parameter $\omega$ is the frequency of oscillations. If the characteristics $T$ and $\mu$ of the model are constant, the Sturm-Liouville problem (1.1) is quite straightforward and the eigenfunctions $u_{n}(z)=$ $\sin (n \pi z / d), n=1,2, \ldots$, form an orthogonal basis of $L^{2}[0, d]$.

In order to consider more general oscillation problems two definitions of relevant Sobolev spaces [1] are recalled.

Definition 1.1. The Sobolev space $H^{k}[0, d]$ is the Hilbert space consisting of all functions $u \in L^{2}[0, d]$ that have generalized derivatives $u^{(j)} \in L^{2}[0, d]$ for each $j=$ $1, \ldots, k$, with the inner product

$$
\left\langle u_{1}, u_{2}\right\rangle_{H^{k}}=\int_{0}^{d} \sum_{j=0}^{k} u_{1}^{(j)} \bar{u}_{2}^{(j)} d z
$$

and corresponding norm $\|u\|_{H^{k}}=\langle u, u\rangle_{H^{k}}^{1 / 2}$.

The complex conjugation of $u$ is denoted by $\bar{u}$. The notation $\left\langle u_{1}, u_{2}\right\rangle$ is used for the standard inner product in $L^{2}[0, d]$, and $\|u\|$ is used for the corresponding norm. In various sections of this work, additional inner products will be introduced to fit the situation being discussed in that section. Each of these inner products will be denoted by $\langle u, v\rangle_{1}$.

Definition 1.2. The Sobolev space $H^{0, k}[0, d]$ is a subspace of $H^{k}[0, d]$ obtained by the closure, in the norm generated by the inner product (1.2), of the set of functions in $H^{k}[0, d]$ that are equal to zero at both end points.

REMARK 1.1. According to the embedding theorems for the Sobolev spaces [1], [6], [7], the functions in $H^{2}[0, d]$ are continuously differentiable on $[0, d]$, though the functions in $H^{1}[0, d]$ can only be assumed to be continuous.

A method is now developed to show that the eigenfunctions for the Sturm-Liouville problem

$$
u^{\prime \prime}+q(z) u=\lambda u, \quad z \in(0, d), \quad u(0)=u(d)=0
$$

form a basis of $L^{2}[0, d]$ (see also [1, p. 169]). The approach used in Section 2 will follow this method. It is assumed that the continuous function $q(z)$ keeps its sign on the interval $[0, d]$. In fact, after the shift of the spectral parameter

$$
\lambda \rightarrow \lambda+Q, \quad Q=\max q(z) \text { for } z \in[0, d],
$$

the function $q(z)$ can be assumed to be negative everywhere on $[0, d]$.

The concept of a generalized solution [1], [6], [7] is fundamental to this work. To derive this concept for system (1.3) multiply the differential equation by the conjugate of an arbitrary $\eta \in H^{0,1}[0, d]$ and integrate by parts over the interval $[0, d]$. Applying the boundary conditions produces the following.

Definition 1.3. The element $u \in H^{0.1}[0, d]$ is called a generalized solution of the Sturm-Liouville problem (1.3) if for any $\eta \in H^{0,1}[0, d]$

$$
-\int_{0}^{d} u^{\prime} \bar{\eta}^{\prime} d z+\int_{0}^{d} q(z) u \bar{\eta} d z=\lambda \int_{0}^{d} u \bar{\eta} d z .
$$

Thus, a solution of the Sturm-Liouville problem (1.3) satisfies (1.4) for all $\eta \in$ $H^{0,1}[0, d]$. To consider the converse, suppose a generalized solution $u$ belongs to $C^{2}[0, d]$. 
Then integrate the first term in the integral identity (1.4) by parts. Since $\eta$ is arbitrary, it follows from the Variational Lemma [8, p. 18] that $u$ will solve the Sturm-Liouville problem (1.3).

Now introduce a new inner product in $H^{0,1}[0, d]$

$$
\langle u, \eta\rangle_{1}=\int_{0}^{d}\left(u^{\prime} \bar{\eta}^{\prime}-q(z) u \bar{\eta}\right) d z
$$

This inner product generates a norm that is equivalent to the standard norm produced by (1.2) for $k=1$. The Riesz representation theorem [1] guarantees that

$$
\int_{0}^{d} u \bar{\eta} d z=\langle A u, \eta\rangle_{1}
$$

for an appropriate linear operator $A: H^{0,1}[0, d] \rightarrow H^{0,1}[0, d]$. The operator $A$ is positive because of the integral definition (1.6). The compactness of $A$ can be shown using the embedding theorems for Sobolev spaces (Sobolev inequalities) [1], [6], [7]. As an example, by using the explicit representation for $A$ in terms of the Green's function for the operator $L=d^{2} / d z^{2}+q(z)$ it can be shown that all properties of $A$ mentioned hold.

Equations (1.4)-(1.6) imply

$$
-\langle u, \eta\rangle_{1}=\lambda\langle A u, \eta\rangle_{1}
$$

for all $\eta \in H^{0,1}[0, d]$. Thus, the above discussion yields the following result for the operator $A$ on the Hilbert space $H^{0,1}[0, d]$, [1, pp. 115, 118].

TheOREM 1.1. The generalized solution $u$ of the Sturm-Liouville problem (1.3) satisfies the equation

$$
-u=\lambda A u
$$

in $H^{0,1}[0, d]$, where $A$ is the positive compact operator on $H^{0,1}[0, d]$ defined by (1.6). This equation has a real, discrete spectrum with the only point of accumulation at infinity. The set of eigenfunctions forms an orthogonal basis of $H^{0,1}[0, d]$.

REMARK 1.2. The density of $H^{0,1}[0, d]$ in $L^{2}[0, d]$ implies that the set of eigenfunctions of (1.7) also forms a basis of $L^{2}[0, d]$. Equations (1.6)-(1.7) imply that this basis is orthogonal in $L^{2}[0, d]$.

The mechanical systems considered in Section 2 have the characteristic property that their eigenoscillations are formulated as a Sturm-Liouville problem with an eigenvalue parameter contained in the boundary conditions. Therefore, the previous classical techniques do not directly apply. In this case the spectral problem (1.7) must be replaced by a problem for an operator polynomial according to the terminology introduced by M. V. Keldysh [9], [10].

In the paper [9], Keldysh considers operator polynomials of an arbitrary order $m$. These operator polynomials will be important in the examples of Section 2. The main idea of [9] is the conversion to a new Hilbert space, denoted by $\Xi^{m}$, which is the orthogonal sum of $m$ copies of the original space $\Xi$. As a result, an equation is obtained in which the spectral parameter appears linearly. In case $m=1$, the simplest operator polynomial 
arises as

$$
L(\lambda) u=0, \quad L(\lambda)=I-T-\lambda V .
$$

Here $I$ is the identity; $T$ and $V$ are operators acting on $\Xi$. This operator polynomial will be central to the results of Section 2 .

Some terminology is needed to state Keldysh's result. Namely, a selfadjoint operator $V$ in $\Xi$ is complete if the equation $V \psi=0$ has only a trivial solution. The operator $V$ has finite order if for its eigenvalues $\lambda_{n}, n=1,2, \ldots$, there exists some finite $p$ such that the following series converges:

$$
\sum_{n \geq 1} \lambda_{n}^{-p}<\infty .
$$

The vectors $u_{n}, n=1,2, \ldots, k$, in $\Xi$ are said to be associated with the eigenvector $u$ defined by $L(\lambda) u=0$ provided that

$$
L(\lambda) u_{r}=V u_{r-1}, \quad r-1, \ldots, k, u_{0}=u .
$$

Theorem 1.2 (Keldysh). If $V$ is a selfadjoint complete operator of finite order and $T$ is a compact operator, then the system of eigenvectors and associated vectors of the spectral problem (1.8) is complete in $\Xi$.

The literature for Sturm-Liouville problems where the spectral parameter appears in the boundary conditions is diverse. The case where the spectral parameter appears linearly in the boundary conditions is discussed first.

Fulton in [11], [12] employed the residue calculus in a manner similar to Titchmarsh [13] to give a direct proof of the convergence properties of the eigenfunction expansion. In [12] the well-known problem of cooling a thin solid bar one end of which is placed in contact with a finite amount of liquid is considered. It is assumed that heat flows from the bar only into the liquid and is convected from the liquid to a surrounding medium; Newton's law of cooling is assumed at the liquid-solid interface. One special case of this problem was considered by Langer [14] (see also Friedman [15]). The general problem was the motivation for [11]-[12], [14]-[16], as well as other authors. Walter [16] obtained the expansion theorem using the selfadjointness of the operator associated with the Sturm-Liouville problem. Note, it is important to emphasize that the Hilbert space $\Xi=L^{2}[0, d] \oplus C$ is used systematically in [11], [12], [15], [16] to get a selfadjoint operator. Bobrovnitskii [17] considered oscillations of some mechanical systems and established the orthogonality relations for eigenfunctions of the corresponding Sturm-Liouville problems with the spectral parameter in the boundary conditions. Those relations contain the terms outside the integral. Note, the structure of functional space mentioned above evidently suggests the presence of these terms. The problem of completeness of eigenfunctions is not discussed in [17]. Hinton [18], [19] considered the same family of Sturm-Liouville problems as Fulton [11], [12], but he included the case of a singular end point. He obtained the convergence theory, uniform and absolute on compact intervals, for the eigenfunction expansions associated with the operator of the SturmLiouville problem. For the case where all eigenvalues of this operator are nonnegative, he proved that the class of functions where convergence takes place is the domain of 
the square root of the operator (energy space). In [20], [21] Hinton and Shaw consider the Sturm-Liouville problem for a vector with the spectral parameter in the boundary conditions. They prove the existence of a Titchmarsh-Weyl coefficient $M(\lambda)$ and use it to construct the Green's function and resolvent operator. The spectrum of the operator is found to be connected with the singular structure of $M(\lambda)$. Hinton and Shaw also give a list of some engineering models where such Sturm-Liouville problems appear. Some of those problems will be mentioned below. Steinrück [22] considered the singular perturbed Sturm-Liouville problem for a couple of functions. He gave an asymptotic analysis of the problem but did not consider completeness of eigenfunctions. Binding and Ye [23] considered the operator polynomial $L(\lambda)=A-\lambda B$, which is similar to (1.8). Here $A$ and $B$ are selfadjoint. The pair $(A, B)$ is simultaneously diagonalizable, in a certain sense, and a variational approach is developed.

The case where the spectral parameter appears nonlinearly in the boundary conditions is more complex. The general results about operator polynomials including the multidimensional case can be found in monographs by Gohberg and Krein [10] and Rodman [24] (see also Radzievskii [25]). Greenberg and Babuska [26], [27] considered Sturm-Liouville problems for second-order and fourth-order differential equations. Here the eigenvalue may occur nonlinearly in the differential operator and in the boundary conditions. Using variational properties of eigenvalues and the Sturm comparison theorems, the authors found bounds for eigenvalues. Among many other papers, we would like to mention only Kostyuchenko and Shkalikov [28], [29] who considered an operator-differential equation in connection with oscillations of an elastic semi-infinite cylinder. They reduced the problem to an operator polynomial equation of the second order

$$
L(\lambda) u=0 \quad \text { with } \quad L(\lambda)=A+\lambda B+\lambda^{2} C .
$$

Here $A$ is bounded and selfadjoint, and $B$ and $C$ are compact and selfadjoint. The authors assume the existence of a constant $c$ such that the operator $L(c)$ is positive and invertible. They proved the completeness of eigenfunctions by studying the resolvent (see [13], [11], [12]). Note, this assumption will be proved for the operator polynomials considered in Section 2. Additional related references from the Russian literature can be found in $[10,25,28,29]$.

Chen et al [30] analyzed the spectrum of two identical coupled beams with dissipative joints. In particular, the asymptotic behavior of the eigenvalues is found. Chen did not consider the problem of the completeness of eigenfunctions. However, the case of dissipation of energy is not considered in this paper. The technique developed here does not apply (at least not directly) to problems where the corresponding operators are not selfadjoint.

The above description shows that diverse experience has been accumulated in operator theory for the proof that a set of eigenfunctions of the Sturm-Liouville problem with the spectral parameter in the boundary conditions forms a basis. Note especially the methods of $[10]$.

The last result to be mentioned deals with Riesz bases [10].

THEOREM 1.3. Every bounded invertible operator transforms any orthonormal basis of $\Xi$ into another basis of $\Xi$, a so-called Riesz basis. 
The main objective of this paper is to prove that the set of eigenfunctions for certain Sturm-Liouville problems arising from mechanics forms a basis for the corresponding space. Note that the Sturm-Liouville problems considered in this work cannot be reduced to the problems considered in [11], [12], [15], [16], though the equations in these references also contain the spectral parameter in boundary conditions. In Section 2, a cable-mass and several beam-mass systems are considered. The main result from abstract operator theory used in that section is Theorem 1.3. The development is such that the associated vectors of Keldysh are not needed in order to obtain a basis of the space $\Xi$. The examples are arranged in order of increasing complexity.

The following result is a direct consequence of the polar identity $[31, \mathrm{p}$. 4]. This inequality is used below in various places to show that an operator is positive.

LEMMA 1.1. $\pm 2 \operatorname{Re}\left(\int u \bar{\eta} d z\right) \geq-\|u\|^{2}-\|\eta\|^{2}$.

2. The eigenfunctions of cable-mass and beam-mass systems. Various cablemass and beam-mass systems are considered in [2]--[5]. However, these authors deal only with eigenfrequencies and their calculations are formal. No existence theorems for eigenfrequencies are proved and no properties of eigenfunctions are discussed except for some numerical results.

In this work, the corresponding Sturm-Liouville problem for each of the cable-mass and beam-mass systems of [2]-[5] is used to define the generalized solution utilizing integral identities. The identity is then reduced to an operator equation in the appropriate Hilbert space. The operator techniques mentioned above are used to prove that the set of eigenfunctions of each system forms a basis of the corresponding Hilbert space. Some estimations for eigenfrequencies from below are found.

2.1. A beam of non-uniform cross section with one end elastically restrained and the other end carrying a guided mass. Bambill and Laura [2] conduct numerical experiments for determining the fundamental frequency of vibration for this system using the Rayleigh-Ritz method.

Such a system is described by the Sturm-Liouville problem

$$
\begin{gathered}
\left(\beta(z) u^{\prime \prime}\right)^{\prime \prime}-\omega^{2} q(z) u=0, \quad z \in(0, d), \\
u^{\prime}(0)=u(d)=0, \quad E I_{0} u^{\prime \prime \prime}(0)=\omega^{2} M u(0), \quad-\Phi E I_{1} u^{\prime \prime}(d)=u^{\prime}(d) .
\end{gathered}
$$

The mechanical parameters $E, I_{0}, I_{1}, \Phi, M$ are assumed to be positive constants that do not contain the spectral parameter $\omega$. The function $\beta(z)$ is assumed to be bounded, measurable, Lebesgue integrable, positive definite on the interval $[0, d]$ and reduces to a (positive) constant near the point $z=0$. Note that $\beta$ was taken to be piecewise constant by Bambill and Laura [2]. The function $q(z)$ is assumed to be bounded, measurable, Lebesgue integrable, and positive on the interval $[0, d]$. The Hilbert space $\Xi$ is taken to be the subspace of functions in $H^{2}[0, d]$ satisfying $u^{\prime}(0)=u(d)=0$. (See Remark 1.1.)

The generalized eigenfunctions of the Sturm-Liouville problem (2.1) in the space $\Xi$ are now defined as in Definition 1.3. (See Remark 1.1.) 
Definition 2.1. The element $u \in \Xi$ is called a generalized solution of the SturmLiouville problem (2.1) if for any $\eta \in \Xi$ the following identity holds:

$$
\begin{aligned}
& \int_{0}^{d} \beta(z) u^{\prime \prime} \bar{\eta}^{\prime \prime} d z+\frac{\beta(d) u^{\prime}(d) \bar{\eta}^{\prime}(d)}{\Phi E I_{1}} \\
& \quad=\omega^{2}\left(\int_{0}^{d} q(z) u \bar{\eta} d z+\frac{M \beta(0) u(0) \bar{\eta}(0)}{E I_{0}}\right) .
\end{aligned}
$$

Now introduce a new inner product on $\Xi$ as follows:

$$
\langle u, \eta\rangle_{1}=\int_{0}^{d} \beta(z) u^{\prime \prime} \bar{\eta}^{\prime \prime} d z+\frac{\beta(d) u^{\prime}(d) \bar{\eta}^{\prime}(d)}{\Phi E I_{1}} .
$$

This inner product generates a norm that is equivalent to the original norm of $u \in \Xi$; see (1.2) for $k=2$.

LEMma 2.1. For the bilinear functional

$$
\Lambda(u, \eta)=\int_{0}^{d} q(z) u \bar{\eta} d z+\frac{M \beta(0) u(0) \bar{\eta}(0)}{E I_{0}}
$$

the following representation holds:

$$
\Lambda(u, \eta)=\langle A u, \eta\rangle_{1}
$$

with a positive compact operator $A$.

The proof is given in Section 3 .

The representation (2.5) and the identity (2.2) result in the generalized eigenfunctions of (2.1) satisfying the following equation in $\Xi$ :

$$
u=\omega^{2} A u .
$$

Theorem 2.1. The eigenfrequencies of the Sturm-Liouville problem (2.1) form a real discrete set with the only point of accumulation at infinity. The system of generalized eigenfunctions forms an orthogonal basis of $\Xi$, and the corresponding eigenfunction expansion converges in the $\Xi$-norm.

Though the spectral parameter $\omega^{2}$ is contained in the boundary conditions $(2.1 \mathrm{~b})$, the final equation (2.6) has the simple structure of (1.7). Theorem 2.1 then follows immediately as in Theorem 1.1 .

Let $\eta=u$ in (2.2) and assume the eigenfunctions are real valued. Then Eq. (2.2) implies that

$$
\begin{aligned}
& \int_{0}^{d} \beta(z)\left(u^{\prime \prime}\right)^{2} d z+\frac{\beta(d) u^{\prime}(d)^{2}}{\Phi E I_{1}} \\
& \quad=\omega^{2}\left(\int_{0}^{d} q(z) u^{2} d z+\frac{M \beta(0) u(0)^{2}}{E I_{0}}\right) .
\end{aligned}
$$

This formula coincides with the formula used by Bambill and Laura [2, Eq. (6)] for numerical calculations of the eigenfrequencies. Using this equation, a Rayleigh-Ritz 
procedure (e.g., see Stakgold [32]) can be developed as

$$
\omega^{2}=\frac{\int_{0}^{d} \beta(z)\left(u^{\prime \prime}\right)^{2} d z+\frac{\beta(d) u^{\prime}(d)^{2}}{\Phi E I_{1}}}{\int_{0}^{d} q(z) u^{2} d z+\frac{M \beta(0) u(0)^{2}}{E I_{0}}} .
$$

2.2. A rotating beam with a tip mass. Transverse vibrations of a rotating beam with a tip mass, such as a helicopter blade or bobbed pendulum, have been considered by many authors (see [33], [34], [35]). By using the notation from [20] the corresponding Sturm-Liouville problem has the form

$$
\begin{gathered}
\left(z^{\prime \prime \prime}-q(x) z^{\prime}\right)^{\prime}=\lambda z, \quad x \in(0,1) \\
z^{\prime \prime}(0)=0, \quad z(1)=z^{\prime}(1)=0, \quad z^{\prime \prime \prime}(0)-c^{2} \gamma^{2} z^{\prime}(0)=\lambda \gamma^{2} z(0) .
\end{gathered}
$$

Here $c$ and $\gamma$ are constants such that $c^{2} \gamma^{2}=q(0)$. The method developed by Hinton and Shaw [20], [21] allows them to include this problem in the general scheme and to get important results about the spectrum. Here the basis property of the eigenfunctions is discussed briefly because the construction is similar to those used in Section 2.1. The generalized solution of the problem $(2.8 \mathrm{a}),(2.8 \mathrm{~b})$ is defined in the space $\Xi$, which is the subspace of functions in $H^{2}[0,1]$ satisfying $z(1)=z^{\prime}(1)=0$. The identity similar to (2.2) has the form

$$
\int_{0}^{1}\left(z^{\prime \prime} \bar{\eta}^{\prime \prime}+q(x) z^{\prime} \bar{\eta}^{\prime}\right) d x=\lambda\left(\int_{0}^{1} z \bar{\eta} d x+\gamma^{2} z(0) \bar{\eta}(0)\right)
$$

The inner product $\langle z, \eta\rangle_{1}$ is introduced as the left-hand side of the identity (2.9). The bilinear functional $\Lambda(z, \eta)$ in the right-hand side can be represented as follows:

$$
\Lambda(z, \eta)=\langle A z, \eta\rangle_{1}
$$

with an appropriate positive linear compact operator $A$. As a result the following equation for the generalized eigenfunctions in $\Xi$ appears:

$$
z=\lambda A z
$$

and the theorem similar to 2.1 holds. The formula for the eigenvalues similar to (2.7) has the form

$$
\lambda=\frac{\int_{0}^{1}\left(\left(z^{\prime \prime}\right)^{2}+q(x)\left(z^{\prime}\right)^{2}\right) d x}{\int_{0}^{1} z^{2} d x+\gamma^{2} z(0)^{2}} .
$$

2.3. A sagged cable supporting a discrete mass. Cheng and Perkins [3] derive the system of equations for an asymptotic model for such a system with small attached mass. A dispersion equation is considered, and frequencies and the shape of the eigenfunctions (modes) are numerically calculated. Additional references related to the problem of behavior of an oscillating cable with tip mass can be found in [20]. In particular, this problem is associated with the feedback control of large flexible space structures [36]. 
Consider the Sturm-Liouville problem

$$
\begin{gathered}
u^{\prime \prime}+\omega^{2} u=s^{2}\left(\int_{0}^{d} u d z+m u\left(z_{0}\right)\right), \quad z \in\left(0, z_{0}\right) \cup\left(z_{0}, d\right), \\
u(0)=u(d)=0, \quad u\left(z_{0}^{+}\right)=u\left(z_{0}^{-}\right) \\
u^{\prime}\left(z_{0}^{+}\right)-u^{\prime}\left(z_{0}^{-}\right)+m \omega^{2} u\left(z_{0}\right)=m s^{2}\left(\int_{0}^{d} u d z+m u\left(z_{0}\right)\right) .
\end{gathered}
$$

The mechanical constants $m$ and $s^{2}$ are positive and do not contain $\omega$.

Definition 2.2. The Hilbert space $\Xi$ consists of all functions $u$ such that $u \in$ $H^{1}\left[0, z_{0}\right], u \in H^{1}\left[z_{0}, d\right], u(0)=u(d)=0$, and $u\left(z_{0}^{+}\right)=u\left(z_{0}^{-}\right)$. (See Remark 1.1.)

Definition 2.3. The element $u \in \Xi$ is the generalized solution of the Sturm-Liouville problem (2.12) if for any $\eta \in \Xi$ the following identity holds:

$$
\begin{aligned}
& \int_{0}^{d} u^{\prime} \bar{\eta}^{\prime} d z+m^{2} s^{2} u\left(z_{0}\right) \bar{\eta}\left(z_{0}\right)+s^{2} \int_{0}^{d} u d z \int_{0}^{d} \bar{\eta} d z \\
& \quad+m s^{2}\left(u\left(z_{0}\right) \int_{0}^{d} \bar{\eta} d z+\bar{\eta}\left(z_{0}\right) \int_{0}^{d} u d z\right)=\omega^{2}\left(\int_{0}^{d} u \bar{\eta} d z+m u\left(z_{0}\right) \bar{\eta}\left(z_{0}\right)\right)
\end{aligned}
$$

Let us introduce the new inner product in $\Xi$ :

$$
\langle u, \eta\rangle_{1}=\int_{0}^{d} u^{\prime} \bar{\eta}^{\prime} d z+m^{2} s^{2} u\left(z_{0}\right) \bar{\eta}\left(z_{0}\right)+s^{2} \int_{0}^{d} u d z \int_{0}^{d} \bar{\eta} d z
$$

and the corresponding norm $\|u\|_{1}=\langle u, u\rangle_{1}^{1 / 2}$, which is equivalent to the original norm (see (1.2) for $k=1$ ).

Lemma 2.2. For the bilinear functionals

$$
\begin{gathered}
\Lambda_{1}(u, \eta)=\int_{0}^{d} u \bar{\eta} d z+m u\left(z_{0}\right) \bar{\eta}\left(z_{0}\right) \\
\Lambda_{2}(u, \eta)=m s^{2}\left(u\left(z_{0}\right) \int_{0}^{d} \bar{\eta} d z+\bar{\eta}\left(z_{0}\right) \int_{0}^{d} u d z\right),
\end{gathered}
$$

the following representations hold:

$$
\Lambda_{k}(u, \eta)=\left\langle A_{k} u, \eta\right\rangle_{1}, \quad k=1,2,
$$

with a positive operator $A_{1}$. Both $A_{1}$ and $A_{2}$ are compact and selfadjoint.

Proof. The proof is discussed in Section 3.

The representations $(2.16)$ and the identity (2.13) result in the identity

$$
\langle u, \eta\rangle_{1}+\left\langle A_{2} u, \eta\right\rangle_{1}=\omega^{2}\left\langle A_{1} u, \eta\right\rangle_{1} .
$$

To summarize, if $u$ is a generalized solution of the Sturm-Liouville problem (2.12), then (2.17) holds for all $\eta \in \Xi$. Therefore, an operator polynomial equation arises instead of the simplest equation (2.6), and the following result is valid. 
Lemma 2.3. The generalized eigenfunctions of the Sturm-Liouville problem (2.12) satisfy the operator equation in $\Xi$

$$
L\left(\omega^{2}\right) u=0, \quad L\left(\omega^{2}\right)=I+A_{2}-\omega^{2} A_{1} .
$$

But, recall that this operator polynomial will not be analyzed using the Keldysh result in Theorem 1.2. Instead, the following result is needed in order to use Theorem 1.3.

Lemma 2.4. The operator polynomial $L\left(-\lambda_{0}\right)$ is positive definite for sufficiently large positive values of $\lambda_{0}$.

Proof. The equations (2.14), (2.15), (2.16), and (2.18) give the formula

$$
\begin{aligned}
\left\langle L\left(-\lambda_{0}\right) u, u\right\rangle_{1}= & \left\|u^{\prime}\right\|^{2}+\left(\lambda_{0} m+m^{2} s^{2}\right)\left|u\left(z_{0}\right)\right|^{2}+s^{2}\left|\int_{0}^{d} u d z\right|^{2} \\
& +2 m s^{2} \operatorname{Re}\left(u\left(z_{0}\right) \int_{0}^{d} \bar{u} d z\right)+\lambda_{0}\|u\|^{2} .
\end{aligned}
$$

Recall that $\|\psi\|$ is the $L^{2}$-norm according to notation in Section 1 . The next estimate follows from Lemma 1.1 by noting that $u\left(z_{0}\right)$ is a constant function,

$$
2 \operatorname{Re}\left(u\left(z_{0}\right) \int_{0}^{d} \bar{u} d z\right) \geq-\|u\|^{2}-d\left|u\left(z_{0}\right)\right|^{2} .
$$

Thus, utilizing (2.14), for sufficiently large positive $\lambda_{0}$,

$$
\begin{aligned}
\left\langle L\left(-\lambda_{0}\right) u, u\right\rangle_{1} \geq & \left\|u^{\prime}\right\|^{2}+\left(\lambda_{0} m+m^{2} s^{2}-m s^{2} d\right)\left|u\left(z_{0}\right)\right|^{2} \\
& +\left(\lambda_{0}-m s^{2}\right)\|u\|^{2}+s^{2}\left|\int_{0}^{d} u d z\right|^{2} \geq\|u\|_{1}^{2} .
\end{aligned}
$$

After the shift of the spectral parameter $\mu=\omega^{2}+\lambda_{0}$ the equation (2.18) has the form

$$
L\left(-\lambda_{0}\right) u=\mu A_{1} u
$$

with a new spectral parameter $\mu$. Now the transformation $v=L\left(-\lambda_{0}\right)^{1 / 2} u$ is introduced in order to apply Theorems 1.1 and 1.3. Here $L\left(-\lambda_{0}\right)^{1 / 2}$ is the positive square root of the positive selfadjoint operator $L\left(-\lambda_{0}\right)[37 ; 38$, p. 60].

LEMMA 2.5. The transformed functions $v=L\left(-\lambda_{0}\right)^{1 / 2} u$ satisfy the operator equation

$$
v=\mu W v, \quad W=L\left(-\lambda_{0}\right)^{-1 / 2} A_{1} L\left(-\lambda_{0}\right)^{-1 / 2},
$$

which for sufficiently large, fixed $\lambda_{0}$ has a positive compact selfadjoint operator $W$. The system of eigenfunctions of this equation forms an orthogonal basis of $\Xi$.

Theorem 2.2. The eigenfrequencies of the Sturm-Liouville problem (2.7) form a real discrete set with the only point of accumulation at infinity. The system of generalized eigenfunctions forms a Riesz basis of $\Xi$, and the corresponding eigenfunction expansion converges in the $\Xi$-norm. 
Proof. The proof follows from Lemma 2.5 by applying Theorem 1.3 to the bounded invertible operator $L\left(-\lambda_{0}\right)^{1 / 2}$. The operator $L\left(-\lambda_{0}\right)^{1 / 2}$ is invertible since it is selfadjoint and the operator $L\left(-\lambda_{0}\right)$ is positive definite and bounded [38, p. 63].

A formula for eigenfrequencies similar to (2.7) can be obtained from Eq. (2.13) as follows:

$$
\omega^{2}=\frac{\int_{0}^{d}\left(u^{\prime}\right)^{2} d z+s^{2}\left(m u\left(z_{0}\right)+\int_{0}^{d} u d z\right)^{2}}{\int_{0}^{d} u^{2} d z+m u\left(z_{0}\right)^{2}} .
$$

2.4. A Timoshenko-Mindlin beam carrying a concentrated mass and a moment of inertia on the end. Maurizi and Bellés ([4], see also [39]) derive the frequency equation for such a system and numerically obtain the natural frequencies of the first three eigenfunctions (modes).

Consider the Sturm-Liouville problem

$$
\begin{gathered}
D \psi^{\prime \prime}-\alpha^{2} G h\left(\psi+\xi^{\prime}\right)+\frac{\rho h^{3}}{12} \omega^{2} \psi=0, \quad z \in(0, d), \\
\alpha^{2} G h\left(\xi^{\prime \prime}+\psi^{\prime}\right)+\rho h \omega^{2} \xi=0, \quad \psi(0)=\xi(0)=0, \\
D \psi^{\prime}(d)-J \omega^{2} \psi(d)=0, \quad \alpha^{2} G h\left(\psi(d)+\xi^{\prime}(d)\right)-M \omega^{2} \xi(d)=0 .
\end{gathered}
$$

The mechanical constants $D, \alpha^{2} G h, \rho, J$, and $M$ are positive and do not contain $\omega$.

Definition 2.4. The Hilbert space $\Xi$ is the subspace of $H^{1}[0, d] \oplus H^{1}[0, d]$ consisting of all two-component functions $\Psi=\left(\begin{array}{l}\psi_{1} \\ \psi_{2}\end{array}\right)$ with $\psi_{1}(0)=\psi_{2}(0)=0$ and inner product

$$
\left\langle\Psi^{(1)}, \Psi^{(2)}\right\rangle_{H^{1}}=\left\langle\psi_{1}^{(1)}, \psi_{1}^{(2)}\right\rangle_{H^{1}}+\left\langle\psi_{2}^{(1)}, \psi_{2}^{(2)}\right\rangle_{H^{1}}
$$

where $\langle u, v\rangle_{H^{1}}$ denotes the standard scalar product (1.2) of $H^{1}[0, d]$.

Definition 2.5. The element $\Psi=\left(\begin{array}{l}\psi_{1} \\ \psi_{2}\end{array}\right)$ is the generalized solution of the SturmLiouville problem (2.20) if for any $\eta=\left(\begin{array}{l}\eta_{1} \\ \eta_{2}\end{array}\right) \in \Xi$ the following identities hold:

$$
\begin{gathered}
D \int_{0}^{d} \psi_{1}^{\prime} \bar{\eta}_{1}^{\prime} d z+\alpha^{2} G h \int_{0}^{d}\left(\psi_{1}+\psi_{2}^{\prime}\right) \bar{\eta}_{1} d z \\
-w^{2}\left(\frac{\rho h^{3}}{12} \int_{0}^{d} \psi_{1} \bar{\eta}_{1} d z+J \psi_{1}(d) \bar{\eta}_{1}(d)\right)=0, \\
\alpha^{2} G h\left(\int_{0}^{d} \psi_{2}^{\prime} \bar{\eta}_{2}^{\prime} d z+\int_{0}^{d} \psi_{1} \bar{\eta}_{2}^{\prime} d z\right) \\
-\omega^{2}\left(\rho h \int_{0}^{d} \psi_{2} \bar{\eta}_{2} d z+M \psi_{2}(d) \bar{\eta}_{2}(d)\right)=0 .
\end{gathered}
$$

Introduce the new inner product in both copies of the space $H^{1}[0, d]$ and in $\Xi$,

$$
\begin{aligned}
\left\langle\psi_{k}, \eta_{k}\right\rangle_{1} & =\int_{0}^{d} \psi_{k}^{\prime} \bar{\eta}_{k}^{\prime} d z, \quad k=1,2, \\
\langle\Psi, \eta\rangle_{1} & =\left\langle\psi_{1}, \eta_{1}\right\rangle_{1}+\left\langle\psi_{2}, \eta_{2}\right\rangle_{1} .
\end{aligned}
$$


Here $\eta=\left(\begin{array}{l}\eta_{1} \\ \eta_{2}\end{array}\right)$. The corresponding norm $\|\Psi\|_{1}=\langle\Psi, \Psi\rangle_{1}^{1 / 2}$ is equivalent to the original norm (see $(2.21))$.

LEMmA 2.6. The following representations are valid:

$$
\begin{aligned}
& \int_{0}^{d} \psi_{k} \bar{\eta}_{k} d z=\left\langle A \psi_{k}, \eta_{k}\right\rangle_{1}, \quad k=1,2, \\
& \int_{0}^{d} \psi_{2}^{\prime} \bar{\eta}_{1} d z=\left\langle B \psi_{2}, \eta_{1}\right\rangle_{1}, \\
& \psi_{k}(d) \bar{\eta}_{k}(d)=\left\langle C \psi_{k}, \eta_{k}\right\rangle_{1}, \quad k=1,2, \\
& \int_{0}^{d} \psi_{1} \bar{\eta}_{2}^{\prime} d z=\left\langle B^{*} \psi_{1}, \eta_{2}\right\rangle_{1}
\end{aligned}
$$

with the positive operators $A$ and $C$. The operators $A, B$, and $C$ are compact. $B^{*}$ here and below denotes an operator conjugate to $B$.

Proof. The proof can be done in the same way as for Lemmas 2.1, 2.2. A direct proof is also possible. For example, an explicit representation for $B$ is

$$
B \psi_{2}=\psi_{2}(d) z-\int_{0}^{z} \psi_{2}(t) d t
$$

Now the compactness of $B$ is clear.

The representations (2.24) and identities (2.22) result in the following operator polynomial.

LEMMA 2.7. The generalized eigenfunctions of the Sturm-Liouville problem (2.20) satisfy the operator equation in $\Xi$,

$$
\begin{gathered}
L\left(\omega^{2}\right) \Psi=0, \quad L\left(\omega^{2}\right)=I+U-\omega^{2} V \\
U=\left(\begin{array}{cc}
a_{1} A & a_{1} B \\
B^{*} & 0
\end{array}\right), \quad V=\left(\begin{array}{cc}
a_{2} A+a_{3} C & 0 \\
0 & a_{4} A+a_{5} C
\end{array}\right)
\end{gathered}
$$

where $a_{1}=\frac{\alpha^{2} G h}{D}, a_{2}=\frac{\rho h^{3}}{12 D}, a_{3}=\frac{J}{D}, a_{4}=\frac{\rho h}{\alpha^{2} G h}, a_{5}=\frac{M}{\alpha^{2} G h}$.

First, the notation is slightly changed. Set $\psi_{2} \rightarrow \kappa \psi_{2}, \kappa=\left(a_{1}\right)^{-1 / 2}$, and divide the second equation by $\kappa$. As a result the operator polynomial equation becomes

$$
L_{1}\left(\omega^{2}\right) \Psi=0, \quad L_{1}\left(\omega^{2}\right)=I+U_{1}-\omega^{2} V, \quad U_{1}=\left(\begin{array}{cc}
a_{1} A & \kappa^{-1} B \\
\kappa^{-1} B^{*} & 0
\end{array}\right) .
$$

The following result is needed in order to use Theorem 1.3.

LEMma 2.8. The operator polynomial $L_{1}\left(-\lambda_{0}\right)$ is a positive-definite operator for all positive values of $\lambda_{0}$.

Proof. The definitions and representations (2.23)-(2.26) result in the formula

$$
\begin{aligned}
\left\langle L_{1}\left(-\lambda_{0}\right) \Psi, \Psi\right\rangle_{1}= & \left\|\psi_{1}^{\prime}\right\|^{2}+\left\|\psi_{2}^{\prime}\right\|^{2}+\left(\lambda_{0} a_{2}+a_{1}\right)\left\|\psi_{1}\right\|^{2}+2 \kappa^{-1} \operatorname{Re}\left\langle\psi_{2}^{\prime}, \psi_{1}\right\rangle \\
& +\lambda_{0} a_{3}\left|\psi_{1}(d)\right|^{2}+\lambda_{0} a_{4}\left\|\psi_{2}\right\|^{2}+\lambda_{0} a_{5}\left|\psi_{2}(d)\right|^{2} .
\end{aligned}
$$


The next estimate follows from Lemma 1.1:

$$
\begin{aligned}
2 \operatorname{Re}\left\langle\psi_{2}^{\prime}, \psi_{1}\right\rangle & =2 \operatorname{Re}\left\langle\varepsilon^{1 / 2} \psi_{2}^{\prime}, \varepsilon^{-1 / 2} \psi_{1}\right\rangle \\
& \geq-\varepsilon\left\|\psi_{2}^{\prime}\right\|^{2}-\varepsilon^{-1}\left\|\psi_{1}\right\|^{2} .
\end{aligned}
$$

Here $\varepsilon$ is an arbitrary positive number. Thus

$$
\begin{gathered}
\left\langle L_{1}\left(-\lambda_{0}\right) \Psi, \Psi\right\rangle_{1} \geq\left\|\psi_{1}^{\prime}\right\|^{2}+\left(1-\kappa^{-1} \varepsilon\right)\left\|\psi_{2}^{\prime}\right\|^{2}+\left(a_{1}+\lambda_{0} a_{2}-\kappa^{-1} \varepsilon^{-1}\right)\left\|\psi_{1}\right\|^{2} \\
+\lambda_{0} a_{3}\left|\psi_{1}(d)\right|^{2}+\lambda_{0} a_{4}\left\|\psi_{2}\right\|^{2}+\lambda_{0} a_{5}\left|\psi_{2}(d)\right|^{2} .
\end{gathered}
$$

Taking $\varepsilon$ in the interval

$$
\kappa>\varepsilon>\kappa^{-1}\left(a_{1}+\lambda_{0} a_{2}\right)^{-1}
$$

yields

$$
\left\langle L_{1}\left(-\lambda_{0}\right) \Psi, \Psi\right\rangle_{1}>\left\|\psi_{1}^{\prime}\right\|^{2}+\left(1-\kappa^{-1} \varepsilon\right)\left\|\psi_{2}^{\prime}\right\|^{2}>\left(1-\kappa^{-1} \varepsilon\right)\|\Psi\|_{1}^{2} .
$$

The inequality (2.27) is true for any $\lambda_{0}>0$ since $a_{2}>0$ implies that $\kappa^{-1}\left(a_{1}+\lambda_{0} a_{2}\right)^{-1}<$ $\kappa^{-1} a_{1}^{-1}=\kappa$. Thus, for any $\lambda_{0}>0$ the operator $L_{1}\left(-\lambda_{0}\right)$ is positive definite.

The proof that the eigenfunctions form a basis of the space can be concluded now utilizing a shift in the spectral parameter and the transformation $\Upsilon=L\left(-\lambda_{0}\right)^{1 / 2} \Psi$, as in Section 2.2.

Theorem 2.3. The eigenfrequencies of the Sturm-Liouville problem (2.20) form a real discrete set with the only point of accumulation at infinity. The system of generalized eigenfunctions forms a Riesz basis of $\Xi$, and the corresponding eigenfunction expansion converges in the $\Xi$-norm.

Assume the eigenfunctions are real valued. Then (2.24), (2.25b), and (2.26) yield

$$
\begin{aligned}
\omega^{2} & =\frac{\left\langle\left(I+U_{1}\right) \Psi, \Psi\right\rangle_{1}}{\langle V \Psi, \Psi\rangle_{1}} \\
& =\frac{\int_{0}^{d}\left(\psi_{1}^{\prime}\right)^{2} d z+\int_{0}^{d}\left(\psi_{2}^{\prime}+\kappa^{-1} \psi_{1}\right)^{2} d z}{a_{2} \int_{0}^{d}\left(\psi_{1}\right)^{2} d z+a_{4} \int_{0}^{d}\left(\psi_{2}\right)^{2} d z+a_{3}\left(\psi_{1}(d)\right)^{2}+a_{5}\left(\psi_{2}(d)\right)^{2}} .
\end{aligned}
$$

2.5. A Timoshenko-Mindlin beam with translational and rotational springs and with a tip mass. Abramovich and Hamburger [5] numerically derive the natural frequencies of such a cantilever Timoshenko beam.

A Sturm-Liouville problem similar to $(2.20)$ is considered. The differential equations are identical but are satisfied for $z \in\left(0, z_{0}\right) \cup\left(z_{0}, d\right)$. The boundary conditions are

$$
\begin{gathered}
\psi(0)=\xi(0)=0, \\
D \psi^{\prime}(d)-J_{1} \omega^{2} \psi(d)+J_{2} \omega^{2} \xi(d)=0, \\
\alpha^{2} G h\left(\psi(d)+\xi^{\prime}(d)\right)-M \omega^{2} \xi(d)+J_{2} \omega^{2} \psi(d)=0 .
\end{gathered}
$$

In addition, the conditions at the interior point $z_{0}$ are

$$
\begin{gathered}
\psi\left(z_{0}^{+}\right)=\psi\left(z_{0}^{-}\right), \quad \xi\left(z_{0}^{+}\right)=\xi\left(z_{0}^{-}\right), \\
D\left(\psi^{\prime}\left(z_{0}^{+}\right)-\psi^{\prime}\left(z_{0}^{-}\right)\right)=k_{r} \psi\left(z_{0}\right), \\
\alpha^{2} G h\left(\xi^{\prime}\left(z_{0}^{+}\right)-\xi^{\prime}\left(z_{0}^{-}\right)\right)=k_{t} \xi\left(z_{0}\right) .
\end{gathered}
$$


The constants $D, \alpha^{2} G h, M, J_{1}, J_{2}, k_{r}, k_{t}$ are positive, do not contain $\omega$, and satisfy the inequality

$$
J_{1} M>J_{2}^{2},
$$

which can be checked easily with the help of formulas from [5]. Other notation for functional spaces, their elements, and operators remains the same as that used in Section 2.4. Except for Lemma 2.11 (below), only a brief description of results and proofs is given since they are similar to those in Section 2.4.

Definition 2.6. The Hilbert space $\Xi$ consists of all two-component functions $\Psi=$ $\left(\begin{array}{l}\psi_{1} \\ \psi_{2}\end{array}\right)$ such that for each $k=1,2, \psi_{k} \in H^{1}\left[0, z_{0}\right], \psi_{k} \in H^{1}\left[z_{0}, d\right]$ with $\psi_{1}(0)=\psi_{2}(0)=0$, and $\psi_{k}$ is continuous at $z_{0}$. The inner product is introduced according to (2.23).

Definition 2.7. The element $\Psi=\left(\begin{array}{l}\psi_{1} \\ \psi_{2}\end{array}\right)$ is a generalized solution of the Sturm-Liouville problem under consideration if for any $\eta=\left(\begin{array}{l}\eta_{1} \\ \eta_{2}\end{array}\right) \in \Xi$ the following identities hold:

$$
\begin{gathered}
D \int_{0}^{d} \psi_{1}^{\prime} \bar{\eta}_{1}^{\prime} d z+\alpha^{2} G h \int_{0}^{d} \psi_{1} \bar{\eta}_{1} d z+\alpha^{2} G h \int_{0}^{d} \psi_{2}^{\prime} \bar{\eta} d z+k_{r} \psi_{1}\left(z_{0}\right) \bar{\eta}_{1}\left(z_{0}\right) \\
=w^{2}\left(\frac{\rho h^{3}}{12} \int_{0}^{d} \psi_{1} \bar{\eta}_{1} d z+J_{1} \psi_{1}(d) \bar{\eta}_{1}(d)-J_{2} \psi_{2}(d) \bar{\eta}_{1}(d)\right) \\
\alpha^{2} G h \int_{0}^{d} \psi_{2}^{\prime} \bar{\eta}_{2}^{\prime} d z+\alpha^{2} G h \int_{0}^{d} \psi_{1} \bar{\eta}_{2}^{\prime} d z+k_{t} \psi_{2}\left(z_{0}\right) \bar{\eta}_{2}\left(z_{0}\right) \\
=\omega^{2}\left(\rho h \int_{0}^{d} \psi_{2} \bar{\eta}_{2} d z+M \psi_{2}(d) \bar{\eta}_{2}(d)-J_{2} \psi_{1}(d) \bar{\eta}_{2}(d)\right)
\end{gathered}
$$

LEMMA 2.9. The following representations are valid:

$$
\psi_{k}\left(z_{0}\right) \bar{\eta}_{k}\left(z_{0}\right)=\left\langle A_{1} \psi_{k}, \bar{\eta}_{k}\right\rangle_{1}, \quad k=1,2,
$$

where the operator $A_{1}$ is positive and compact.

Lemma 2.10. The generalized eigenfunctions of the Sturm-Liouville problem under consideration satisfy the operator equation in $\Xi$ :

$$
\begin{gathered}
L\left(\omega^{2}\right) \Psi=0, \quad L\left(\omega^{2}\right)=I+U-\omega^{2} V \\
U=\left(\begin{array}{cc}
a_{1} A+a_{6} A_{1} & a_{1} B \\
B^{*} & a_{7} A_{1}
\end{array}\right), \quad V=\left(\begin{array}{cc}
a_{2} A+a_{8} C & -a_{9} C \\
-a_{10} C & a_{4} A+a_{5} C
\end{array}\right),
\end{gathered}
$$

where $a_{6}=\frac{k_{r}}{D}, a_{7}=\frac{k_{t}}{\alpha^{2} G h}, a_{8}=\frac{J_{1}}{D}, a_{9}=\frac{J_{2}}{D}, a_{10}=\frac{J_{2}}{\alpha^{2} G h}$, and the operators and other constants are given in Lemmas 2.6 and 2.7.

Substituting $\psi_{2} \rightarrow \kappa \psi_{2}, \kappa=\left(a_{1}\right)^{-1 / 2}$ and dividing the second equation by $\kappa$, yields the following equation:

$$
L_{1}\left(\omega^{2}\right) \Psi=0, \quad L_{1}\left(\omega^{2}\right)=I+U_{1}-\omega^{2} V_{1} .
$$

Here, instead of $U$ and $V$ as in (2.25), the selfadjoint operators are

$$
U_{1}=\left(\begin{array}{cc}
a_{1} A+a_{6} A_{1} & \kappa^{-1} B \\
\kappa^{-1} B^{*} & a_{7} A_{1}
\end{array}\right), \quad V_{1}=\left(\begin{array}{cc}
a_{2} A+a_{8} C & -\kappa_{1} C \\
-\kappa_{1} C & a_{4} A+a_{5} C
\end{array}\right)
$$


with $\kappa_{1}=a_{9} \kappa=a_{10} \kappa^{-1}$, since $a_{1}=a_{9} / a_{10}$.

LEMMA 2.11. The operator polynomial $L_{1}\left(-\lambda_{0}\right)$ is a positive-definite operator for all positive values of $\lambda_{0}$.

Proof. The beginning of the proof coincides with the proof of Lemma 2.8 (recall that $\langle u, v\rangle$ is in the inner product of $L^{2}[0, d]$ with norm $\left.\|u\|\right)$ :

$$
\begin{aligned}
\left\langle L_{1}\left(-\lambda_{0}\right) \Psi, \Psi\right\rangle_{1}= & \left\|\psi_{1}^{\prime}\right\|^{2}+\left\|\psi_{2}^{\prime}\right\|^{2}+\left(a_{1}+\lambda_{0} a_{2}\right)\left\|\psi_{1}\right\|^{2}+\lambda_{0} a_{4}\left\|\psi_{2}\right\|^{2} \\
& +a_{6}\left|\psi_{1}\left(z_{0}\right)\right|^{2}+a_{7}\left|\psi_{2}\left(z_{0}\right)\right|^{2}+\lambda_{0} a_{8}\left|\psi_{1}(d)\right|^{2}+\lambda_{0} a_{5}\left|\psi_{2}(d)\right|^{2} \\
& -2 \kappa_{1} \lambda_{0} \operatorname{Re}\left(\psi_{2}(d) \bar{\psi}_{1}(d)\right)+2 \kappa^{-1} \operatorname{Re}\left\langle\psi_{2}^{\prime}, \psi_{1}\right\rangle .
\end{aligned}
$$

The next estimates follow from Lemma 1.1:

$$
\begin{aligned}
2 \operatorname{Re}\left\langle\psi_{2}^{\prime}, \psi_{1}\right\rangle & =2 \operatorname{Re}\left\langle\varepsilon^{1 / 2} \psi_{2}^{\prime}, \varepsilon^{-1 / 2} \psi_{1}\right\rangle \\
& \geq-\varepsilon\left\|\psi_{2}^{\prime}\right\|^{2}-\varepsilon^{-1}\left\|\psi_{1}\right\|^{2} \\
-2 \operatorname{Re}\left(\psi_{2}(d) \bar{\psi}_{1}(d)\right) & =-2 \operatorname{Re}\left(\varepsilon_{1}^{1 / 2} \psi_{2}(d) \varepsilon_{1}^{-1 / 2} \psi_{1}(d)\right) \\
& \geq-\varepsilon_{1}\left|\psi_{1}(d)\right|^{2}-\varepsilon_{1}^{-1}\left|\psi_{2}(d)\right|^{2} .
\end{aligned}
$$

Here $\varepsilon$ and $\varepsilon_{1}$ are two arbitrary positive numbers. Using (2.34), yields

$$
\begin{aligned}
\left\langle L_{1}\left(-\lambda_{0}\right) \Psi, \Psi\right\rangle_{1} \geq & \left\|\psi_{1}^{\prime}\right\|^{2}+\left(1-\kappa^{-1} \varepsilon\right)\left\|\psi_{2}^{\prime}\right\|^{2}+\left(a_{1}+\lambda_{0} a_{2}-\kappa^{-1} \varepsilon^{-1}\right)\left\|\psi_{1}\right\|^{2} \\
& +\lambda_{0} a_{4}\left\|\psi_{2}\right\|^{2}+a_{6}\left|\psi_{1}\left(z_{0}\right)\right|^{2}+a_{7}\left|\psi_{2}\left(z_{0}\right)\right|^{2} \\
& +\lambda_{0}\left(a_{8}-\kappa_{1} \varepsilon_{1}\right)\left|\psi_{1}(d)\right|^{2}+\lambda_{0}\left(a_{5}-\kappa_{1} \varepsilon_{1}^{-1}\right)\left|\psi_{2}(d)\right|^{2} .
\end{aligned}
$$

Let us take $\varepsilon$ in the interval (2.27) and $\varepsilon_{1}$ in the interval

$$
a_{8} \kappa_{1}^{-1}>\varepsilon_{1}>\kappa_{1} a_{5}^{-1}
$$

As was mentioned in the proof of Lemma 2.8, the inequalities (2.27) do not impose any restrictions on the positive parameters $a_{1}, a_{2}$. The choice in (2.35) of the parameter $\varepsilon_{1}$ is possible if $a_{5} a_{8}>\kappa_{1}^{2}$. The representations of all these parameters (see Lemmas 2.7 and 2.10) and the inequality (2.30) show that this inequality is true. This finally yields

$$
\left\langle L_{1}\left(-\lambda_{0}\right) \Psi, \Psi\right\rangle_{1}>\left(1-\kappa^{-1} \varepsilon\right)\|\Psi\|_{1}^{2} .
$$

Theorem 2.4. The eigenfrequencies of the Sturm-Liouville problem (2.20) under boundary conditions (2.29) form a real discrete set with the only point of accumulation at infinity. The system of generalized eigenfunctions forms a Riesz basis of $\Xi$, and the corresponding eigenfunction expansion converges in the $\Xi$-norm. 
The following formula for eigenfrequencies, which is similar to (2.28), can be obtained from Eqs. (2.24), (2.32), (2.33), and Lemma 2.9:

$$
\begin{aligned}
\omega^{2} & =\frac{\left\langle\left(I+U_{1}\right) \Psi, \Psi\right\rangle_{1}}{\left\langle V_{1} \Psi, \Psi\right\rangle_{1}} \\
& =\frac{\int_{0}^{d}\left(\left(\psi_{1}^{\prime}\right)^{2}+\left(\psi_{2}^{\prime}\right)^{2}\right) d z+a_{1} \int_{0}^{d}\left(\psi_{1}\right)^{2} d z+a_{6}\left(\psi_{1}\left(z_{0}\right)\right)^{2}+a_{7}\left(\psi_{2}\left(z_{0}\right)\right)^{2}+\frac{2}{\kappa} \int_{0}^{d} \psi_{2}^{\prime} \psi_{1} d z}{a_{2} \int_{0}^{d}\left(\psi_{1}\right)^{2} d z+a_{8}\left(\psi_{1}(d)\right)^{2}+a_{4} \int_{0}^{d}\left(\psi_{2}\right)^{2} d z+a_{5}\left(\psi_{2}(d)\right)^{2}-2 \kappa_{1} \psi_{1}(d) \psi_{2}(d)} \\
& =\frac{\int_{0}^{d}\left(\psi_{1}^{\prime}\right)^{2} d z+a_{6}\left(\psi_{1}\left(z_{0}\right)\right)^{2}+a_{7}\left(\psi_{2}\left(z_{0}\right)\right)^{2}+\int_{0}^{d}\left(\psi_{2}^{\prime}+\kappa^{-1} \psi_{1}\right)^{2} d z}{\int_{0}^{d}\left(a_{2}\left(\psi_{1}\right)^{2}+a_{4}\left(\psi_{2}\right)^{2}\right) d z+a_{8}\left(\psi_{1}(d)\right)^{2}-2 \kappa_{1} \psi_{1}(d) \psi_{2}(d)+a_{5}\left(\psi_{2}(d)\right)^{2}} .
\end{aligned}
$$

Recall that $a_{1}=\kappa^{-2}$ and that $a_{8} a_{5}>\left(\kappa_{1}\right)^{2}$ from (2.30). Thus, the denominator is positive.

2.6. Estimations for eigenfrequencies from below. The formulas (2.7), (2.19), (2.28), and (2.36) allow the estimation of lower bounds for the eigenfrequencies

$$
\omega \geq \omega_{*}
$$

This estimation for problems in Sections 2.1 and 2.3 is now derived explicitly in terms of mechanical parameters of the corresponding systems. Such estimations exist for the problems in Sections 2.4 and 2.5 but are sufficiently more complex to derive. The corresponding estimation for the problem in Section 2.2 is similar to those in 2.1 .

For the beam (Section 2.1) the derivation is based on the representation (2.7) and the inequalities

$$
\begin{aligned}
\int_{0}^{d} q(z) u^{2} d z & \leq C_{1}\langle u, u\rangle_{1} \\
u(0)^{2} & \leq C_{2}\langle u, u\rangle_{1}
\end{aligned}
$$

for appropriate constants $C_{1}$ and $C_{2}$, which are independent of the function $u(z), u \in \Xi$. Recall that the inner product is given by (2.3). Combining (2.7), (2.38), and (2.39) yields

$$
\omega^{2} \geq\left(C_{1}+\frac{M \beta(0) C_{2}}{E I_{0}}\right)^{-1} .
$$

The inequalities (2.38), (2.39) are just particular cases of the embedding theorems for the Sobolev space $H^{2}[0, d]$ (e.g., see [1], [6], [7]). The functions from this space are continuously differentiable on $[0, d]$. The goal is to find the explicit representation for $C_{1}$ and $C_{2}$. Using the second boundary condition (2.1b) one finds

$$
u(z)=\int_{d}^{z} u^{\prime}(t) d t
$$

The Schwarz Inequality yields

$$
u(z)^{2}=\left(\int_{d}^{z} u^{\prime}(t) d t\right)^{2} \leq(d-z) \int_{z}^{d}\left(u^{\prime}(t)\right)^{2} d t
$$


for any $z \in[0, d]$. Further, with help of the first of the boundary conditions (2.1b) it follows that

$$
u^{\prime}(z)=\int_{d}^{z} u^{\prime \prime}(t) d t
$$

Arguments similar to those mentioned above yield

$$
\begin{aligned}
\left(u^{\prime}(z)\right)^{2} & =\left(\int_{0}^{z} \frac{1}{\sqrt{\beta(t)}}\left(\sqrt{\beta(t)} u^{\prime \prime}(t)\right) d t\right)^{2} \\
& \leq \int_{0}^{z} \frac{1}{\beta(t)} d t \int_{0}^{z} \beta(t)\left(u^{\prime \prime}(t)\right)^{2} d t \\
& \leq \int_{0}^{z} \frac{1}{\beta(t)} d t\langle u, u\rangle_{1} .
\end{aligned}
$$

Therefore,

$$
\int_{z}^{d}\left(u^{\prime}(t)\right)^{2} d t \leq \int_{z}^{d} \int_{0}^{t} \frac{1}{\beta(\tau)} d \tau d t\langle u, u\rangle_{1}
$$

Combining inequalities (2.42) and (2.44) yields

$$
\begin{aligned}
u(z)^{2} & \leq(d-z) \int_{z}^{d} \int_{0}^{t} \frac{1}{\beta(\tau)} d \tau d t\langle u, u\rangle_{1} \\
& =(d-z) \int_{0}^{d} y(\tau, z) \frac{1}{\beta(\tau)} d \tau\langle u, u\rangle_{1}
\end{aligned}
$$

where the function $y(\tau, z)=d-\max (z, \tau)$ is introduced. In particular, inequality (2.39) holds with

$$
C_{2}=d \int_{0}^{d} \frac{d-\tau}{\beta(\tau)} d \tau
$$

Further, inequality (2.46) shows that

$$
\int_{0}^{d} q(z) u^{2} d z \leq \int_{0}^{d} q(z)(d-z) \int_{0}^{d} \frac{y(\tau, z)}{\beta(\tau)} d \tau d z\langle u, u\rangle_{1}
$$

and, therefore, the inequality (2.38) holds with

$$
C_{1}=\int_{0}^{d} q(z)(d-z) \int_{0}^{d} \frac{y(\tau, z)}{\beta(\tau)} d \tau d z
$$

Combining (2.37), (2.40), (2.47), and (2.49) yields

$$
\omega_{*}=\left\{\int_{0}^{d} q(z)(d-z) \int_{0}^{d} \frac{y(\tau, z)}{\beta(\tau)} d \tau d z+\frac{M \beta(0) d}{E I_{0}} \int_{0}^{d} \frac{d-\tau}{\beta(\tau)} d \tau\right\}^{-1 / 2} .
$$

Note that this estimation does not depend on the parameter $\Phi E I$. The following inequality is a simpler, but more rough, estimate:

$$
\frac{1}{\beta(\tau)} \leq\left(\min _{[0, d]} \beta(z)\right)^{-1} .
$$


Finally,

$$
\omega \geq \omega_{*}=\sqrt{2 \min _{[0, d]} \beta(z)}\left\{\int_{0}^{d} q(z)(d-z)\left(d^{2}-z^{2}\right) d z+\frac{M \beta(0) d^{3}}{E I_{0}}\right\}^{-1 / 2} .
$$

For the cable (Section 2.3) similar arguments to those used above produce the inequalities

$$
\begin{aligned}
\int_{0}^{d} u^{2} d z & \leq C_{3}\langle u, u\rangle_{1}, \quad C_{3}=\frac{d^{2}}{2}, \\
u\left(z_{0}\right)^{2} & \leq C_{4}\langle u, u\rangle_{1}, \quad C_{4}=\min \left(z_{0}, d-z_{0}\right)
\end{aligned}
$$

where the inner product is given by (2.14). The inequality (2.53) follows from (2.42) by integration, and the inequality (2.54) follows from (2.42). Finally,

$$
\omega \geq \omega_{*}=\left\{\frac{d^{2}}{2}+m \min \left(z_{0}, d-z_{0}\right)\right\}^{-1 / 2} .
$$

Note, this estimation does not depend on the cable parameter $s$.

REMARK 2.1. The results of this paper can be further developed in three directions. First, the formulas (2.7), (2.19), (2.28), and (2.36) allow the development of variational techniques for the numerical analysis of eigenfunctions and eigenfrequencies [32]. This approach is especially important when the parameters of the system are not constant. Second, the spectral analysis of the above mechanical systems is the first step in the development of a theory of control for those models. In particular, the asymptotic representation of eigenfrequencies for large numbers is important in such an analysis. Third, the corresponding eigenfunction expansions allow the consideration of the oscillations of systems under the action of random forces [40]. Current research on these three topics by the authors uses the techniques and results developed in this paper.

REMARK 2.2. After this work was complete the authors became aware of the work of Laura, Reyes, and Rossi [41] on cable-payload systems. The corresponding SturmLiouville problem contains a spectral parameter in the boundary conditions and presents a sixth example to which the technique developed in this paper can be applied. The methods of Fulton $[11,12]$ and Hinton $[18,19]$ can also be applied to this system.

\section{Proofs of compactness.}

1. The proof of Lemma 2.1. Recall, the Hilbert space $\Xi$ is taken to be the subspace of functions in $H^{2}[0, d]$ satisfying $u^{\prime}(0)=u(d)=0$ (see Remark 1.1) and the inner product is given by $(2.3)$. The functional $\Lambda(u, \eta)$ allows the following obvious estimate:

$$
|\Lambda(u, \eta)| \leq c_{1}\|u\|\|\eta\|+c_{2}|u(0)||\eta(0)| .
$$

Here and below, $c_{k}(k=1,2, \ldots)$ denote different positive constants whose exact values are not important for the proof.

The inequalities (2.38) and (2.39) imply

$$
\begin{aligned}
\|u\| & \leq c_{3}\|u\|_{1}, \\
|u(0)| & \leq c_{4}\|u\|_{1} .
\end{aligned}
$$


Hence, the functional $\Lambda(u, \eta)$ allows the following estimate:

$$
|\Lambda(u, \eta)| \leq c_{5}\|u\|_{1}\|\eta\|_{1} .
$$

The estimate (3.4) shows that $\Lambda(u, \eta)$ is a linear functional in $\eta \in \Xi$ for any given $u \in \Xi$. The Riesz representation theorem leads to the representation (2.5) with a bounded linear operator $A: \Xi \rightarrow \Xi$. The selfadjointness of $A$ and its positivity are obvious because the function $q(z)$ is positive and bounded and the parameter $c_{2}=M \beta(0) /\left(E I_{0}\right)$ is positive (see (2.4)). Now the compactness of the operator $A$ will be proven [6]. Let the sequence $\left\{u_{k}\right\}$ converge weakly in $\Xi$ to an element $u \in \Xi$. It will be shown that the sequence $\left\{A u_{k}\right\}$ converges strongly in $\Xi$. The boundedness of $A$ implies the weak convergence of $\left\{A u_{k}\right\}$ to $A u$ in $\Xi$. Moreover, the compactness of the embedding operator of $\Xi$ into $L^{2}[0, d][6]$ implies the strong convergence of the sequences $\left\{u_{k}\right\}$ and $\left\{A u_{k}\right\}$ in $L^{2}[0, d]$ to $u$ and $A u$, respectively. Further, the compactness of the embedding operator of $\Xi$ into $C[0, d][6]$ implies the strong convergence of the sequences $\left\{u_{k}(0)\right\}$ and $\left\{\left(A u_{k}\right)(0)\right\}$ in $\mathbb{C}$ to $u(0)$ and $(A u)(0)$, respectively. The representation (2.5) and the inequality (3.1) imply

$$
\begin{aligned}
\left\|A\left(u_{k}-u_{m}\right)\right\|_{1}^{2} & =\Lambda\left(u_{k}-u_{m}, A\left(u_{k}-u_{m}\right)\right) \\
& \leq c_{1}\left\|u_{k}-u_{m}\right\|\left\|A\left(u_{k}-u_{m}\right)\right\|+c_{2}\left|\left(u_{k}-u_{m}\right)(0)\right|\left|\left(A\left(u_{k}-u_{m}\right)\right)(0)\right| \\
& \rightarrow 0 \quad \text { as } k, m \rightarrow \infty
\end{aligned}
$$

Hence, the sequence $\left\{A u_{k}\right\}$ converges strongly in $\Xi$. The compactness of the operator $A$ is proven.

2. The proof of Lemma 2.2. Recall, the Hilbert space $\Xi$ consists of all functions $u$ such that $u \in H^{1}\left[0, z_{0}\right], u \in H^{1}\left[z_{0}, d\right], u(0)=u(d)=0$, and $u\left(z_{0}^{+}\right)=u\left(z_{0}^{-}\right)$(see Remark $1.1)$ and the inner product is given by (2.14).

The functional $\Lambda_{2}(u, \eta)$ allows the following bound:

$$
\left|\Lambda_{2}(u, \eta)\right| \leq c_{6}\left(\left|u\left(z_{0}\right)\right| \int_{0}^{d}|\eta| d z+\left|\eta\left(z_{0}\right)\right| \int_{0}^{d}|u| d z\right) .
$$

Hölder's inequality implies

$$
\left|\Lambda_{2}(u, \eta)\right| \leq c_{7}\left(\left|u\left(z_{0}\right)\right|\|\eta\|+\left|\eta\left(z_{0}\right)\right|\|u\|\right) .
$$

The inequality (3.7) and the inequalities (2.53), (2.54) imply

$$
\left|\Lambda_{2}(u, \eta)\right| \leq c_{8}\|u\|_{1}\|\eta\|_{1} .
$$

The further proof is essentially the same as for Lemma 2.1. As a result, the representation (2.16) is justified for $k=2$. The proof for the case $k=1$ is easier and does not differ essentially from the proof for Lemma 2.1 .

Acknowledgments. The authors would like to thank Professor Donald Hinton, University of Tennessee, for his helpful remarks. The first author would like to thank Professor P. A. A. Laura, Institute of Applied Mechanics, Argentina, for suggesting this work. 


\section{REFERENCES}

[1] J. P. Keener, Principles of Applied Mathematics: Transformation and Approximation, AddisonWesley Publishing Company, Redwood City, California, 1988

[2] E. A. Bambill and P. A. A. Laura, Application of the Rayleigh-Schmidt method when the boundary conditions contain the eigenvalues of the problem, Journal of Sound and Vibration 130, 167-170 (1989)

[3] S.-P. Cheng and N. C. Perkins, Free vibration of a sagged cable supporting a discrete mass, Journal of the Acoustical Society of America 91, 2654-2662 (1992)

[4] M. J. Maurizi and P. M. Bellés, An additional evaluation of free vibrations of beam-mass systems, Journal of Sound and Vibration 154, 182-186 (1992)

[5] H. Abramovich and O. Hamburger, Vibration of a uniform cantilever Timoshenko beam with translational and rotational springs and with a tip mass, Journal of Sound and Vibration 154, 67-80 (1992)

[6] O. A. Ladyzhenskaia, The Boundary Value Problems of Mathematical Physics, Springer-Verlag, New York, New York, 1985

[7] D. Gilbarg and N. S,. Trudinger, Elliptic Partial Differential Equations of Second Order, 2nd edition, Springer-Verlag, Berlin, 1983

[8] E. Zeidler, Nonlinear Functional Analysis and its Applications, Part II/A, Linear Monotone Operators, Springer-Verlag, New York, New York, 1990

[9] M. V. Keldysh, On the characteristic values and characteristic functions of a certain class of nonselfadjoint equations, Dokl. Akad. Nauk SSSR 77, 11-14 (1951) (in Russian)

[10] I. C. Gohberg and M. G. Krein, Introduction to the Theory of Linear Non-Selfadjoint Operators, Translation of Mathematical Monographs, Vol. 18, American Mathematical Society, Providence, Rhode Island, 1969

[11] C. T. Fulton, Singular eigenvalue problems with eigenvalue parameter contained in the boundary conditions, Proceedings of the Royal Society of Edinburg 87A, 1-34 (1980)

[12] C. T. Fulton, Two-point boundary value problems with eigenvalue parameter contained in the boundary conditions, Proceedings of the Royal Society of Edinburg 77 A, 293-308 (1977)

[13] E. C. Titchmarsh, Eigenfunction Expansions Associated with Second Order Differential Equations, I, 2nd edition, University Press, Oxford, London, 1962

[14] R. E. Langer, A problem in diffusion or in the flow of heat for a solid in contact with a fluid, Tôhoku Mathematics Journal 35, 360-375 (1932)

[15] B. Friedman, Principles and Techniques of Applied Mathematics, John Wiley and Sons, New York, New York, 1956

[16] J. Walter, Regular eigenvalue problems with eigenvalue parameter in the boundary conditions, Math. Zeitschrift 133, 301-312 (1973)

[17] Yu. I. Bobrovnitskii, On oscillations of some mechanical systems with nonorthogonal eigenfunctions, Acoustic Dynamics of Machines and Constructions "Nauk", Moscow, Russia, 1973, pp. 6-9 (in Russian)

[18] D. B. Hinton, An expansion theorem for an eigenvalue problem with eigenvalue parameter in the boundary condition, Quarterly Journal of Mathematics, Oxford, Vol. 2, 33-42 (1979)

[19] D. B. Hinton, Eigenfunction expansions for a singular eigenvalue problem with eigenvalue parameter in the boundary condition, SIAM Journal of Mathematical Analysis 12, 572-584 (1981)

[20] D. B. Hinton and J. K. Shaw, Differential Operators with Spectral Parameter Incompletely in the Boundary Conditions, Funkcialaj Ekvacioj (Serio Internacia), Vol. 33, 1990, pp. 363-385

[21] D. B. Hinton and J. K. Shaw, Spectrum of a Hamiltonian System with Spectral Parameter in a Boundary Condition, Canadian Mathematical Society Proceedings 8, 171-186 (1987)

[22] H. Steinrück, Singularly perturbed eigenvalue problems, SIAM Journal of Applied Mathematics 47, 1131-1149 (1987)

[23] P. Binding and Q. Ye, Variational principles without definiteness conditions, SIAM Journal of Mathematical Analysis 22, 1575-1583 (1991)

[24] L. Rodman, An Introduction to Operator Polynomials, Birkhäuser-Verlag, Boston, Massachusetts, 1989

[25] G. V. Radzievskii, A problem of completeness of root vectors in the spectral theory of operatorfunctions, Uspechi of Mathematical Sciences 37, 81-145 (1982) (in Russian) 
[26] L. Greenberg and I. Babuska, A continuous analogue of Sturm sequences in the context of SturmLiouville equations, SIAM Journal of Numerical Analysis 26, 920-945 (1989)

[27] L. Greenberg, An oscillation method for fourth-order, self-adjoint, two-point boundary value problems with nonlinear eigenvalues, SIAM Journal of Mathematical Analysis 22, 1021-1042 (1991)

[28] A. G. Kostyuchenko and A. A. Shkalikov, Self-adjoint quadratic operator pencils and elliptic problems, Functional Analysis and its Applications 17, 109-128 (1983)

[29] A. G. Kostyuchenko and A. A. Shkalikov, On the theory of self-adjoint quadratic operator polynomials, Vestnik Moscov. University Series 1. Mathematics, Mechanics, No. 6, 1983, pp. 40-51 (in Russian)

[30] G. Chen, S. G. Krantz, D. L. Russell, C. E. Wayne, H. H. West, and M. P. Coleman, Analysis, design and behavior of dissipative joints for coupled beams, SIAM Journal of Applied Mathematics 49, 1665-1693 (1989)

[31] J. B. Conway, A Course in Functional Analysis, Springer-Verlag, New York, New York, 1985

[32] I. Stakgold, Boundary Value Problems of Mathematical Physics, Volume II, Macmillan Co., New York, New York, 1971

[33] H. J. Ahn, Vibrations of a pendulum consisting of a bob suspended from a wire, Quart. Appl. Math. 39, 109-117 (1981)

[34] H. J. Ahn, On random transverse vibrations of a rotating beam with tip mass, Quart. Appl. Math. 39, 97-109 (1981)

[35] L. H. Jones, The transverse vibration of a rotating beam with tip mass, Quart. Appl. Math. 23, 193-203 (1975)

[36] J. A. Burns and E. M. Cliff, An approximation technique for the control and identification of the hybrid system, in Dynamics and Control of Large Flexible Space Structures, Meirovich, Virginia Tech, Blacksburg, VA, 1981, pp. 269-284

[37] F. Riesz and B. St. Nagy, Lectures in Functional Analysis, Ungar, New York, New York, 1955

[38] W. Schmeidler, Linear Operators in Hilbert Space, Academic Press, New York, New York, 1965

[39] R. D. Mindlin, Influence of rotatory inertia and shear on flexural motions of isotropic, elastic plates, Journal of Applied Mechanics 18, A31-A38 (1951)

[40] Z. Schuss, Theory and Applications of Stochastic Differential Equations, 1980

[41] P. A. Laura, J. A. Reyes, and R. E. Rossi, Dynamic behavior of a cable-payload system suddenly stopped at one end, Journal of Sound and Vibration 34, 81-95 (1989) 